\title{
About the Universe
}

\author{
Angel Fierros Palacios \\ Instituto de Investigaciones Eléctricas, División de Energías Alternas, Cuernavaca Morelos, México \\ Email: afierros@iie.org.mx
}

How to cite this paper: Palacios, A.F. (2017) About the Universe. Open Access Library Journal, 4: e3223.

https://doi.org/10.4236/oalib.1103223

Received: January 28, 2017

Accepted: May 14, 2017

Published: May 17, 2017

Copyright @ 2017 by author and Open Access Library Inc.

This work is licensed under the Creative Commons Attribution International License (CC BY 4.0).

http://creativecommons.org/licenses/by/4.0/

\begin{abstract}
In this paper, it is proposed that the size of the classical electron, which is a stable elemental particle with the smallest concentration of matter in Nature, can be used to explain the very big size of the Universe. In order to reach that objective, the apparent size of heavenly bodies as seemed each other at very big distances in space, is used as a fundamental concept. Also, it is proved that the size, shape, mass, and future of the Universe are ruled by the speed of light, and the range of gravitational interactions.
\end{abstract}

\section{Subject Areas \\ Classical Mechanics}

\section{Keywords}

Relativistic Red Shift, the Apparent Size of the Cosmic Egg, Cosmic Geometry, Size, Mass, Mean Mass Density of the Universe, Greatest Velocity in the Universe, Range of Gravitational Interactions

\section{Introduction}

According to Einstein's Special Theory of Relativity, the speed of light in vacuum is the upper limit of the velocity of any heavenly body. However, it is well known that it is a velocity which cannot be reached by any kind of matter. In other words, no material body could possibly move with a velocity equal or greater than the speed of light. The only particle which can travel at speed of light is the photon. This fact means that no heavenly body could possibly reach distances equal or greater than those reached by photons. That natural limitation points out a limit of the size of the Universe. On the other hand, the speed of gravitational interactions must be considered as equal to the escape velocity due to the apparent size of the source of gravity; which depends on its mass and critically on its apparent radius [1]. It is the medium used by gravity to transmit its effects like propagating force of Nature [1]. Therefore, it is possibly to be sure 
that the maximum range of gravitational interactions is equal to the maximum radius of the Universe. Moreover, gravity is related to the shape, and size of the Universe, if it is consider the only force that governs its evolution, and also its future [2]; because it is capable of stopping the expansion, and turn it into a contraction [2]. The maximum range of gravitational interactions is giving by the escape velocity due to the apparent size of the Cosmic Egg as seen from the edge of the Universe. For that reason, it is proposed that the apparent size of the Cosmic Egg could be equal to the size of the classical electron. That argument points towards the fact that both sizes are intimately related.

As it is well known, the Cosmos is expanding for a long time, and this motion is still visible at present. Any theory about the Universe must account for this expansion; and also for the observed fact that it looks the same in every direction. If it follows the expansion backward in time, it is easy to see that the Universe must have been smaller in the past. There was a time when all the matter and energy was packed tightly together in a relatively small region of space, currently called the Primeval Atom or also the Cosmic Egg [2]. This primeval atom then exploded in the most fantastic explosion called by G. Gamow the Big Bang, flinging all the heavenly bodies off into space.

The Big Bang Theory sent all the fragments of the Cosmic Egg moving away from one another. It was established that the more distant a galaxy, the greater in direct proportion, is its velocity of recession as determined by the shifts of its spectral lines to the red wavelengths. The velocities of recession of those galaxies are proportional to their distances. This relationship is now known as the Law of red shifts, or also Hubble's Law [3]. Anyway, if the Universe is open, it will go expanding forever, whereas if it is closed the expansion will slow to stop, and then will become a contraction [2]. The Universe that stops expanding, is a closed Universe and the ever expanding one is an open Universe [2].

\section{The Relativistic Red Shift}

The red shift of any heavenly body is usually denoted by the letter $z$, that is to say

$$
z=\frac{\Delta \lambda}{\lambda}
$$

where $\Delta \lambda$ is the red shift in a wavelength of any spectral line, and $\lambda$ the wavelength when it left the body [4]. The red shifts of quasars range from relatively small numbers to large [4]. The red shift can also be expressed as a velocity by means of the Doppler Shift formula. If the speed of the body is small compared to the speed of light, the following simple form of that formula is used

$$
\frac{v}{c}=\frac{\Delta \lambda}{\lambda}
$$

where $v$ is the velocity of recession of the body, and $c$ the speed of light. It is known that quasars have very large red shifts, showing that they are moving at relativistic velocities. Then, it is necessary to use the following exact relativistic Doppler Shift relationship [4] 


$$
Z=\frac{(1+v / c)^{1 / 2}}{(1-v / c)^{1 / 2}}-1 .
$$

From this equation, one gets that

$$
Z=\frac{z^{2}+2 z}{z^{2}+2 z+2}
$$

where $z$ is the red shift measured from the spectra [4]. Let

$$
Z=\frac{v}{c}
$$

be the relativistic red shift. Clearly, $Z<1$. Thus, $v<c$ always. According to $H u b$ ble's LaW

$$
v=H r
$$

where $H$ is Hubble's constant. Hence, from Equation (5) it is obtained that

$$
H=\frac{Z c}{r} .
$$

From that relationship, it is possible to compare any couple of quasars, and consider one of them as a measure unit, to obtain that [4]

$$
\frac{Z_{1}}{Z_{o}}=\frac{r_{1}}{r_{o}}
$$

where $Z_{o}$ and $r_{o}$ are the relativistic red shift and the distance respectively, of the quasar unit, as long as $Z_{1}$, and $r_{1}$ are the relativistic red shift of the other quasar. Then, to calculate extragalactic distances, only is needed the red shift measured directly from the respective spectra, and use Equation (4) to obtain the following relation [4]

$$
r=\frac{r_{o} Z}{Z_{o}} .
$$

Let us consider the quasar $3 C 273$ as a unit. Its red shift measured from the spectrum is equal to $z=0.158$. From Equation (4) the corresponding relativistic red shift is $Z_{o}=0.1457$. The nearest large cluster of galaxies is the Virgo Cluster, 23.9 Megaparsecs distant. The velocity of recession of that cluster is equal to $1200 \mathrm{~km} \cdot \mathrm{sec}^{-1}$. Given that this velocity is small compared to the speed of light, Equation (2) is used to obtain that $z=0.4 \times 10^{-2}$. Then, using the data of that cluster; the parameters of the unit quasar are the following [4]

$$
\begin{aligned}
& Z_{o}=0.1457 \\
& r_{o}=871 \text { Megaparsecs. }
\end{aligned}
$$

\section{The Cosmic Space Scale}

According to Einstein's Special Theory of Relativity, no material particle could possibly travel with a velocity greater than the speed of light. It became obvious that nobody could be accelerated beyond the light barrier [5]. This argument still stands. All the heavenly bodies have a proper mass different from zero. Then, they can only move by means of an acceleration process. That theory said that 
the speed of light is the upper limit of the velocities of all the heavenly bodies. This argument points out towards a closed universe model [5]. Consider a closed, spherical Universe. It is well known that the surface of a sphere has no edge. However, in a spherical Universe, it is possible to assume that it has a size with a limit. Then, using the methodology before given, it can be estimate the distance from the edge of the size limit to the Cosmic Egg.

The quasar $O Q 172$ is the most distant quasar known at this time. It has a red shift measured from the spectrum equal to $Z=3.53$. From Equation (4) the relativistic red shift is $Z=0.9071$. Also, form Equation (5) the velocity of recession is $V=272,130 \mathrm{~km} \cdot \mathrm{sec}^{-1}$; and the distance to the Cosmic Egg is equal to $r=5421$ Megaparsecs.

To estimate the distance between the edges of the size limit to the Cosmic Egg, let us suppose that there is a heavenly body near that edge. The velocity of recession of that hypothetic body will be $v=299,625 \mathrm{~km} \cdot \mathrm{sec}^{-1}$; which correspond to the relativistic red shift $Z=0.98875$. That body is $r=5911$ Megaparsecs distant to the Cosmic Egg. According to all what it was said before, it is proposed that the maximum radius of the spherical Universe is equal to $r_{u}=6 \times 10^{3}$ Megaparsecs.

\section{Cosmic Geometry}

The shape of the Cosmos is related to its future if gravity is assumed to be the only force governs its evolution [2]. Also, it is important to take into account the results of Einstein's Special Theory of Relativity [5], about the speed of light as the upper limit, before mentioned.

If it is considered that the shape of the Universe is an sphere, must have a radius equal to

$$
r_{u}=1.85 \times 10^{28} \mathrm{~cm} ;
$$

and a volume

$$
V_{u}=2.64 \times 10^{85} \mathrm{~cm}^{3} .
$$

So that it is important to determine, how many heavenly bodies can be contained in that volume. To solve the problem is necessary to know the mass of the Universe, and also its mean mass density to determine its shape.

\section{The Mean Mass Density of the Universe}

The Universe contains many types of objects: Galaxies, clusters of galaxies, etc. there may also be intergalactic gas, and other bodies like quasars, and black holes [2]. All of them must be taken into account to estimate its total mass.

Let us consider some kind of regions whose mass must be greater to the mass of a typical galaxies like our galaxy. The Milky Way is a disk galaxy, about $5 \times$ $10^{4}$ light years in radius and $3.26 \times 10^{2}$ light years thick. The disk is composed of spiral arms which are a characteristic feature of other spiral galaxies. It is known that it contain $2 \times 10^{11}$ solar masses [2].

Let us consider that every galaxy and also certain amount of matter from the 
other heavenly bodies, are packed in the regions before mentioned, which have spherical shape with a radius equal to

$$
r=6.8 \times 10^{20} \mathrm{~cm} .
$$

The volume of each region is

$$
V=1.3 \times 10^{63} \mathrm{~cm}^{3} \text {. }
$$

Then, from Equations (12) and (14) the number of those regions contained in the universal volume is given by

$$
\text { Number of regions }=2 \times 10^{22} \text {. }
$$

Hence, the amount of mass contained in the Universe is given by the product of the solar mass and the number of those regions; that is to say

$$
M_{u}=4 \times 10^{55} \text { gr. }
$$

The mean mass density required to make a closed Universe is called the closed density; since is the critical density needed to stop the expansion and turn it into a contraction. From Equations (12) and (16) one gets that

$$
\rho_{u}=1.5 \times 10^{-30} \mathrm{gm} \cdot \mathrm{cm}^{-3} .
$$

\section{The Size of the Universe}

As it was proved before by means of the relativistic red shift, the Universe is not infinite in space, but certainly it has a very big size which is determined by the distance travel by the heavenly bodies at a velocity smaller than the speed of light [5]. The gravity is the only force which determines the structure of the Universe, and also governs its evolution and future. In other words, gravity transmits its effects like a propagating force of Nature; so that, is one of dynamic agents that determines the size of the Universe. Let us consider the following argumentation.

When a couple of heavenly bodies separated by a distance in space interact each other, as it was said before, their apparent sizes as seemed at a distance, play a special role [5]; because the values of their escape velocities are dependent on their mass, and critically on their apparent radius [1]. It can be proved that the escape velocity due to the apparent size of the bodies is the medium used by gravity to transmit its effects at a distance in space [1]. That velocity is such that increases its value with the distance.

In order to determine the very big size of the Universe, the concept of apparent size can be used. Let us consider an observer on a heavenly body which is near the limit of the Universe. At such a distance, it is practically impossible to see the Cosmic Egg. However, that object has an apparent size. It is proposed that its apparent size is equal to the classical electron size; which has the following classical radius [6]

$$
r_{e}=2.82 \times 10^{-13} \mathrm{~cm} .
$$

Consider that value as the apparent radius of the Cosmic Egg in the relation- 
ship for the escape velocity due to the apparent size [5]; that is to say

$$
v_{\max }=\left(\frac{2 G M_{u}}{r_{e}}\right)^{1 / 2},
$$

where $M_{u}$ is the total mass of the Universe. The Belgian cleric Abbé G. Lemaitre make the suggestion in 1925 that in a remote past that total mass were concentered in the Cosmic Egg. Also, in Equation (19) it has that

$$
G=6.66 \times 10^{-8} \mathrm{ergs} \cdot \mathrm{cm} \cdot \mathrm{gr}^{-2},
$$

is the Gravitational Constant. Hence,

$$
v_{\max }=4.35 \times 10^{25} \mathrm{~km} \cdot \mathrm{sec}^{-1} .
$$

This is the maximum velocity which can be reached in the Universe. It is the medium used by gravity to transmit its effects at a very big distance in space. Clearly,

$$
v_{\max }=v_{u}=v_{g} .
$$

In a Dynamic Theory of Gravitation [1], it is considered that $v_{g}$ is the fundamental velocity [1].

Finally, that velocity travels the distance between the Cosmic Egg and the edge of the limit size of the Universe in the time given by

$$
\tau_{u}=0.43 \times 10^{-2} \mathrm{sec} .
$$

This is the minimum transit time taken by the velocity of the gravitational interactions to travel at a distance equal to the Cosmos radius.

\section{Conclusions}

The relativistic red shift was used to prove that the Universe has a finite and maximum size. This is so because of the value of the speed of light in vacuum. This upper limit of the velocities of heavenly bodies is valid for any kind of matter. That argument points towards an escape velocity of the Universe equal or greater than the speed of light.

Also, as it was said before, the Big Bang sent all the fragments of the Cosmic Egg flinging off into space, with an initial speed called the velocity of recession. At present, all of them could possibly travel at a very low speed. Given that the gravity force is always attractive, it is possibly to assume that the gravitational interactions play a special role in the size and structure of the Universe.

On the other hand, the mean mass density is referred as the closed critical density. Its value generally accepted is about $10^{-30} \mathrm{gr} \cdot \mathrm{cm}^{-3}$; which is of the same order of magnitude to the value obtained in the text. So that, if the Universe is closed, it is a hyperspherical Universe.

Finally, it is proposed the concept of apparent size due to the very big distances in space can be used to show that the size of the Universe, and the size of the classical electron, are closely related; that is to say, the very big size of the Universe is determined by the small size of the classical electron. 


\section{References}

[1] Fierros Palacios, A. (2016) Gravitation. Open Access Library Journal, 3, e2141. https://doi.org/10.4236/oalib.1102141

[2] Shipman, H.I. (1976) Black Holes. Quasars, and the Universe. Houghton Mifflin Company, Boston.

[3] Fierros, A. (2016) Hubble's Constant. Journal of High Energy Physics, Gravitation and Cosmology, 2, 293-297. https://doi.org/10.4236/jhepgc.2016.23025

[4] Fierros, A. (2016) About the Quasars. Journal of High Energy Physics, Gravitation and Cosmology, 2, 323-327. https://doi.org/10.4236/jhepgc.2016.23030

[5] Fierros, A. (2016) The Missing Mass Problem. Journal of High Energy Physics, Gravitation and Cosmology, 2, 298-300. https://doi.org/10.4236/jhepgc.2016.23026

[6] Leighton, R.B. (1959) Principles of Modern Physics. McGraw-Hill Company, New York, Toronto, London.

\section{Appendix}

The mean mass density method really shows that the Universe is closed [2]. According to specialized literature the value of the critical density required to make a closed Universe is about $10^{-30} \mathrm{gr} \mathrm{cm}^{-3}$; or one hydrogen atom in a volume of space the size of a desk [2]. That is to say, that volume is equal to $10^{6} \mathrm{~cm}^{3}$. Therefore, the volume $V_{u}$ contains

$$
2.67 \times 10^{79} \text { hydrogen atoms. }
$$

The mass of the hydrogen atom is [6]

$$
m_{H}=1.67 \times 10^{-24} \mathrm{gr} .
$$

So that

$$
M_{u}=4.4 \times 10^{55} \mathrm{gr}
$$

which is the same value given in Equation (16), obtained by means of the methodology proposed in the text. Clearly, it doesn't need much mass to close the Universe. It is quite empty [2]. 
Submit or recommend next manuscript to OALib Journal and we will provide best service for you:

- Publication frequency: Monthly

- 9 subject areas of science, technology and medicine

- Fair and rigorous peer-review system

- Fast publication process

- Article promotion in various social networking sites (LinkedIn, Facebook, Twitter, etc.)

- Maximum dissemination of your research work

Submit Your Paper Online: Click Here to Submit

Or Contact service@oalib.com 